\title{
Intrahepatic Injection of Adenovirus Reduces Inflammation and Increases Gene Transfer and Therapeutic Effect in Mice
}

Julien Crettaz, ${ }^{1}$ Pedro Berraondo, ${ }^{1}$ Itsaso Mauleón, ${ }^{1}$ Laura Ochoa, ${ }^{1}$ Vijay Shankar, ${ }^{2}$ Miguel Barajas, ${ }^{1}$ Nico van Rooijen, ${ }^{3}$ Stefan Kochanek, ${ }^{2}$ Cheng Qian, ${ }^{1}$ Jesús Prieto, ${ }^{1}$ Rubén Hernández-Alcoceba, ${ }^{1}$ and Gloria González-Aseguinolaza ${ }^{1}$

Recombinant adenoviruses (Ad) are among the most extensively used vectors for liver gene transfer. One of the major limitations for the clinical application of these vectors is the inflammatory immune response associated with systemic administration of high dose of virus. We evaluated the effect of Ad administration route on the inflammatory immune response and liver transgene expression. We compared direct intrahepatic injection (IH) with the systemic administration via tail vein (IV). IH injection of Ad resulted in a lower inflammatory response and a higher transgene expression. When a relatively low dose of virus was used, IV administration resulted in no detectable protein expression but production of proinflammatory cytokines. In contrast, IH administration induced high levels of transgene expression and no inflammation, although we detected a transient hypertransaminemia, which fully resolved within days. Furthermore, IH injection resulted in a faster protein expression being more intense at the site of injection, whereas IV administration caused slower but diffuse liver expression. IH injection also reduced the spreading of the virus to other organs. Independently of the route, depletion of Kupffer cells significantly enhanced the transduction efficiency of Ad. This effect was stronger when using IV injection, indicating that IH injection partially overcomes Kupffer cell phagocytic activity. Moreover, the antitumor efficacy of high-capacity-Ad encoding murine interleukin-12 (IL-12) was significantly greater when the vector was administered by IH injection than when given IV. In conclusion, IH injection of adenovirus represents a safe and efficient administration route for clinical applications of gene therapy targeting the liver. (HEPATOLOGY 2006;44:623-632.)

Abbreviations: Ad, adenoviruses; HC, high-capacity; IL, interleukin; TNF- $\alpha$, tumor necrosis factor alpha; IFN, interferon; $I V$, intravenous; $I H$, intrahepatic; $L M L$, left medial lobe; PBS, phosphate-buffered saline; AST, aspartate aminotransferase; $A L T$, alanine aminotransferase; p fu, plaque-forming units; iu, infection units.

From the ${ }^{1}$ Division of Gene Therapy and Hepatology, Center for Applied Medical Research (CIMA), University of Navarra, Pamplona, Spain; the ${ }^{2}$ Division of Gene Therapy, University of Ulm, Ulm, Germany; and the ${ }^{3}$ Department of Cell and Immunology, Faculty of Medicine, Vrije Universiteit Medical Center, Amsterdam, The Netherlands.

Received March 30, 2006; accepted June 2, 2006.

Dr. Berraondo is currently affiliated with Unité de Biologie des Régulations Immunitaires, INSERM E 352, Institut Pasteur, Paris, France. Dr. Barajas is currently affliated with Division of Hematology, Department of Medicine, University of Minnesota, Minneapolis, $M N$.

Supported by grants UTE project CIMA, Instituto Salud Carlos III C03/02 and PI051098 and SAF 2002-0327 (Ministerio Educación y Ciencia) to J.P., G. G-A and P. B. and Department of Education and Culture of the Government of Navarra (IIQ4273). Julien Crettaz was in receipt of a grant from Gobierno de Navarra. Pedro Berraondo was in receipt of grant 009175 from FIS (Ministerio de Salud). Laura Ochoa was in receipt of a grant from Fundación Areces. Vijay Shankar was in receipt of a grant from Sander Foundation.

Address reprint requests to: Dra Gloria González-Aseguinolaza, Centro de Investigación Médica Aplicada (CIMA), Terapia Génica de Hepatitis Virales, Avda Pío XII 55, 31008 Pamplona, Spain.E-mail: ggasegui@unav.es; fax: (34) 948194717.

Copyright (C) 2006 by the American Association for the Study of Liver Diseases.

Published online in Wiley InterScience (www.interscience.wiley.com).

DOI 10.1002/hep.21292

Potential conflict of interest: Nothing to report.
A denoviral vectors have been shown to efficiently transfer genetic material to the liver in many experimental models and clinical trials. ${ }^{1-3}$ First-generation adenoviruses (Ad) present several advantages over other gene therapy vectors reviewed in Breyer et al. ${ }^{4}$ and Vorburger and Hunt. ${ }^{5}$ Recently, Ad vectors that are devoid of all viral coding sequences, called gutless or highcapacity adenovirus (HC-Ad), have been developed. The absence of viral genes minimizes the immune response against the virus, provides large cloning capacity, and enables long-term transgene expression. ${ }^{6,7}$ Although these vectors have substantial advantages with respect to firstgeneration adenoviruses, the production process of HC-Ad is very laborious, and the virus particles can still stimulate immune responses, probably as a result of particle-receptor interaction or internalization. ${ }^{89}$ The major limitation of adenoviral vectors resides in the strong inflammatory reaction generated by the intravascular injection of a high number of viral particles. ${ }^{10,11}$ In fact, infusion of a high dose of second-generation E1/E4 deleted adenovirus into the hepatic artery of a patient with partial ornithine transcarbamylase deficiency was fatal because of a massive inflammatory response. ${ }^{12,13}$ Adenoviral vectors are predominantly sequestered by the liver within 
minutes after their intravenous administration. A substantial proportion of the vector dose is captured by Kupffer cells, and only a fraction of them infect hepatocytes, establishing a threshold of the viral dose that should be administered to obtain detectable amounts of the transgene product. ${ }^{14-18}$ Furthermore, on adenovirus uptake, Kupffer cells get activated and release cytokines, such as interleukin-6 (IL-6), IL-12, IL-10, IL-8, tumor necrosis factor alpha (TNF- $\alpha$ ), interferon gamma (IFN$\gamma$ ), and chemokines such as MIP- $1 \alpha$ and MIP-2. ${ }^{19,20}$ Moreover, viral particles are processed by Kupffer cells that act as antigen-presenting cells activating helper $\mathrm{T}$ cells and cytotoxic $\mathrm{T}$ lymphocytes, which eliminate the infected cells. ${ }^{21}$ Therefore, gene therapy procedures using adenovirus vectors should make use of strategies aiming at circumventing macrophage phagocytic activity to reduce toxicity and enhance hepatocyte transduction efficiency.

In this work we attempted to improve adenoviral gene transfer to the liver by simply modifying the route of vector delivery. Although intravenous (IV) administration is the simplest way to transduce the liver, other organs such as the spleen, heart, and kidney are also transduced. ${ }^{22}$ Intraportal and intra-arterial administration does not appear to be different from peripheral intravenous injection with respect to efficiency of hepatic transduction, systemic vector dissemination, or toxicity. ${ }^{23,24}$ Biliary infusion is an alternative, but it is technically difficult and may cause complications. ${ }^{25}$ Another route of administration frequently used for adenovirus administration in cancer gene therapy protocols is the intratumoral injection. This route has been shown to be well tolerated and to reduce the spread of the virus. ${ }^{26,27}$ Our study compares the standard intravenous administration with direct injection of the virus into liver parenchyma (intrahepatic injection, IH) using first-generation adenovirus and high-capacity adenoviral vectors. This approach has been used for in utero adenoviral administration but has not been characterized in adult animals. ${ }^{28}$

Our results indicate that intrahepatic injection of either first-generation or high-capacity adenoviruses increased liver transgene expression and reduced the inflammatory reaction by circumventing liver macrophages. Also we show that the improvement of liver transduction leads to enhanced antitumoral activity of the vector encoding murine IL-12.

\section{Materials and Methods}

Animals. Six- to 8-week-old BALB/c female mice (Taconic Laboratories, Germantown, NY) were used in all studies except tumor experiments, in which C57BL/6J mice were used. Animal handling was performed accord- ing to regulations of the local animal commission. Bleeding was realized by retroorbital sinus blood extraction, and serum was collected for subsequent analysis.

Adenovirus Vectors. E1- and E3-deleted adenoviral vectors carrying human IL-12, luciferase, and $\beta$-galactosidase cDNA were constructed as previously described. ${ }^{27,29}$ High-capacity or gutless adenovirus vectors encoding human IL-12 (HC-Ad/RUhIL-12) or murine IL-12 (HC-Ad/RUmIL-12) were obtained as described. ${ }^{30}$ Adenoviruses at different doses were administered intravenously (through the tail vein in a total volume of 200 $\mu \mathrm{L})$ and intrahepatically, after anesthesia (ketamine), through direct injection in the left medial lobe (LML) in a volume of $50 \mu \mathrm{L}$.

Macrophage Depletion by Clodronate-Loaded Liposomes. Liposome-encapsulated clodronate (clodronate-liposomes) and liposomes containing phosphatebuffered saline (PBS) (control liposomes) were prepared as previously described. ${ }^{31,32}$ Dichloromethylene-biphosphonate (or clodronate) was a kind gift of Roche Diagnostics Gmbh, Mannheim, Germany. Clodronateliposomes and PBS-liposomes were administered intravenously by the tail vein at a dose of $20 \mu \mathrm{L} / 10 \mathrm{~g}$ animal weight in a total volume of $50 \mu \mathrm{L}$ in saline buffer.

Histological Analysis. Total depletion of liver macrophages was confirmed in each experiment by immunofluorescence staining using the F4/80 antibody and with a FITC-labeled goat anti-rat secondary antibody (Pierce Biotechnology Inc., Rockford, IL). The immunofluorescence signal was quantified by the Aquacosmos software (Hamamatsu Photonics). X-gal histochemistry was performed on OCT-embedded frozen tissues. Sections were cut in a cryostat and fixed in $0.5 \%$ glutaraldehyde at room temperature for 10 minutes. After removal the glutaraldehyde the preparations were immersed in X-gal solution and mounted in glycerol.

Determination of Adenovirus Genome Particles in Different Tissues. DNA was isolated from frozen tissues using the DNeasy Tissue Kit (Qiagen, Hilden, Germany) following the manufacturer's instructions. Adenovirus DNA was quantified by means of real-time quantitative polymerase chain reaction. Primers and TaqMan probe (Applied Biosystems, Foster City, CA) for Adenovirus E4 gene were designed using the Primer-Express software. GAPDH was quantified by real-time quantitative polymerase chain reaction as described. ${ }^{33}$ Results were expressed as viral genomes $(\mathrm{vg})$ per $10^{-6}$ genome copies of GAPDH.

Determination of Serum Levels of Aspartate Aminotransferase and Alanine Aminotransferase. The blood level of aspartate aminotransferase (AST) and alanine aminotransferase (ALT) were measured using com- 
mercial kits (Sigma Chemicals, St. Louis MO) in an Hitachi Automatic Analyzer (Boehringer Mannheim, Indianapolis, IN).

Determination of Serum Levels of bIL-12 and Murine $I L-12, I L-6$, and IFN- $\gamma$. Sera were assayed at a 1:10 dilution for the hIL-12, mIL- 6 , and mIFN- $\gamma$ and at a 1:50 dilution for the mIL-12 using OptEIA ELISA Set kits (Pharmingen, San Diego CA) according to the manufacturer's protocol.

In Vivo Macroscopic Imaging of Luciferase Activity. Mice were anesthetized and received a $100-\mu \mathrm{L}$ dose of D-Luciferin Firefly (Xenogen, Alameda, CA) intraperitoneally. Mice were placed in a luminometric in vivo system, a cooled charged coupled device Xenogen IVIS, and data were analyzed with a Living Image Software (Xenogen). Areas of $3 \mathrm{~cm}$ diameter were quantified in photons per second after background subtraction. ${ }^{34}$

Luciferase Measurement. Tissue sections were frozen in liquid nitrogen until processed. Tissue was homogenized in a Luciferase Lysis Reagent (Promega, Madison, WI). Samples were centrifuged for 15 seconds at $12,000 \mathrm{~g}$. Supernatant was collected and measured in a tube luminometer. Total proteins were quantified by Bradford assay using bovine serum albumin as a standard.

Tumor Model. Liver metastases were produced by injecting $5 \times 10^{5} \mathrm{MC} 38$ cells in C57BL/6J mice into the LML after laparotomy in $50 \mu \mathrm{L}$ saline. Survival was checked daily, and animals were killed if moribund.

Statistical Analysis. All calculations were performed with SPSS 11.0 software. A normality test (KolmogorovSmirnov) was used to analyze all samples. Normally distributed samples were analyzed by a paired ANOVA test for transgene expression experiments. Biodistribution, inflammation, and depletion assays were analyzed by a Student $t$ test. The data are presented as mean \pm SD. Nonnormally distributed samples of the cytokine analysis in the tumor study were analyzed by a Mann-Whitney test and presented as median (25th, 75th percentile) in the box-plot. Tumor volume studies were analyzed by a Kruskal-Wallis test and $2 \times 2$ comparisons by a MannWhitney test. Each group of the survival study was analyzed by the Kaplan-Meier method, and groups were compared by a log-rank test. $P$-value was considered statistically significant when $P<.05\left(^{*}\right)$ and very significant when $\left.P<.01{ }^{(* *}\right)$.

\section{Results}

Effect of the Route of Adenovirus Administration on Liver Transduction Efficiency. We used a first-generation adenovirus encoding human IL-12 (AdhIL12) to study the effect of the route of administration on liver transduction. AdhIL12 was administered to groups of six $\mathrm{BALB} / \mathrm{c}$ mice using two different routes, intravenous injection (IV) and intrahepatic injection (IH) and two doses, $10^{9}$ and $10^{10}$ plaque-forming units (pfu) per mouse. Serum samples were obtained at days 0 (basal), 1, 2,3 , and 7 after the injection, and hIL-12 was analyzed by ELISA. As shown in Fig. 1B, transgene expression was significantly higher after $\mathrm{IH}$ administration of $10^{10} \mathrm{pfu}, 1$ and 2 days after injection than after IV administration ( $P=.031$ and $P=.042$ respectively); however, values were similar from day 3 onward. More importantly, IH administration of $10^{9}$ pfu AdhIL-12 (Fig. 1A) resulted in hIL12 serum levels similar to those obtained after IV injection of a dose 10 times higher $\left(10^{10} \mathrm{pfu}\right)(1,972.8 \pm$ $1,309.9 \mathrm{pg} / \mathrm{mL}$ vs $2,670.8 \pm 2,811 \mathrm{pg} / \mathrm{mL})$, whereas in mice given $10^{9}$ pfu AdhIL-12 via IV route no hIL12 was detected.

To further confirm our observations, we analyzed the pattern and intensity of expression of a first-generation recombinant adenovirus encoding luciferase (Adluc). BALB/c mice were injected IH and IV with $10^{9}$ and $10^{10} \mathrm{pfu} / \mathrm{mouse}$. Luciferase expression was determined and quantified at days $1,3,7$, and 10. As shown in Fig. 1C-D, significant differences on the level of transgene expression were observed at days 1 and 3 between IH and IV injected animals $(P=.001)$; the differences disappeared after day 7. As in the case of AdhIL12, the expression level of $10^{9} \mathrm{pfu}$ of Adluc given by IH route was similar to the level obtained after IV injection of a dose 10-fold higher.

We also found differences in the kinetics of transgene expression depending on the route. Whereas the peak of IL-12 expression was observed 72 hours after IV administration, the highest expression level of hIL-12 after IH injection was detected at 24 hours (Fig. 1B). In the case of Adluc, transgene expression peaked at 24 hours after virus injection, whereas after IV injection the peak was at day 7 (Fig. 1C-D).

Next we asked whether IH injection would also improve liver transduction using a high-capacity adenovirus vector. For this purpose a mifepristone-regulable highcapacity (gutless) adenovirus encoding hIL12 (HC-Ad/ RUhIL-12) was administered to BALB/c mice $(n=6)$ by IV or IH injection at a dose of $10^{\circ}$ infection units (iu) per mouse. Two weeks after the administration of the virus, the inducer, RU486, was administered intraperitoneally at a dose of $250 \mu \mathrm{g} / \mathrm{kg}$ animal weight. Serum was extracted before RU486 administration and 4, 10, 24, and 48 hours after, and protein expression was analyzed by ELISA. As shown in Fig. 1E, significantly higher levels of protein expression were observed after IH injection 10 

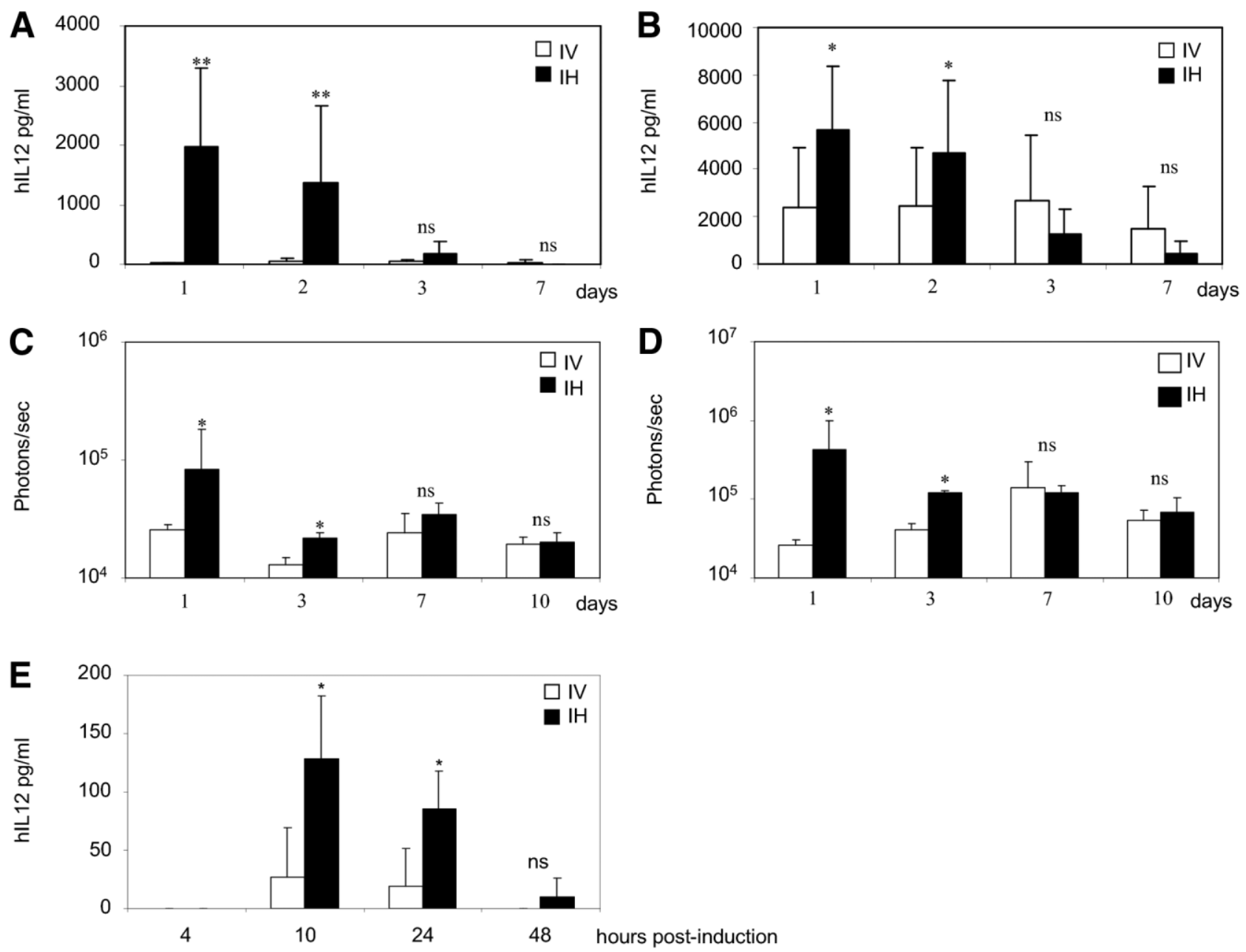

Fig. 1. Improvement of liver transduction by intrahepatic injection of first-generation and high-capacity adenovirus. BALB/c mice were injected with AdhlL12 by intrahepatic (IH) or intravenous (IV) route at a dose of $10^{9}$ (A) plaque-forming units (pfu) or $10^{10}$ (B) pfu/mouse. Human IL-12 concentration in serum was measured. BALB/c mice were injected with Adluc IH or IV at a dose of $10^{9}$ (C) pfu or $10^{10}$ (D) pfu/mouse. Luciferase expression was analyzed and quantified. (E) BALB/c mice were injected with $10^{9}$ infection units (iu)/mouse of HC-Ad/RUhlL-12 by IV or IH injection. Two weeks after the administration of the virus, mifepristone was administered intraperitoneally at a dose of $250 \mu \mathrm{g} / \mathrm{kg}$. Human IL-12 expression in serum is presented. All data are presented as a mean of the values obtained from six mice. Error bars represent \pm SD of luciferase and hIL-12 data from six mice. Data from one of two experiments are provided $\left({ }^{*} P<.05 * * P<.01\right)$.

and 24 hours after the administration of the inducer $(P=$ .034 and $P=.038$, respectively).

Analysis of Virus Biodistribution. The analysis of luciferase expression using the charged coupled device camera showed that the mice that received the vector intravenously showed a diffuse expression of the transgene throughout the whole liver, whereas those animals that were injected by the $\mathrm{IH}$ route showed a local expression at the site of vector injection (Fig. 2A). To confirm and supplement in vivo data imaging, animals were sacrificed 24 hours after the administration of $10^{10}$ pfu Adluc or AdLacZ, liver was excised, and the LML, right medial lobe, and right lateral lobe were separated. In both cases transgene expression was mainly detected at the injected lobe after IH injection, whereas transgene expression was similar in the three lobes after IV administration, indicat- ing that, by this route, the transduction events were distributed uniformly throughout the liver (Fig. 2B). More importantly the analysis of luciferase expression in other organs such as the spleen indicated a lower spreading of the virus after IH injection (Fig. 2B). To further corroborate these data, the biodistribution of the vector was analyzed by quantification of viral genomes in different organs 24 hours after adenovirus injection. As shown in Fig. 2D, IH administration clearly reduces the presence of adenovirus in lung, heart, and spleen, in comparison with IV injection. Furthermore, in agreement with the expression analysis, we observed that after IH injection the amount of virus is significantly higher in the injected lobe.

The Systemic Inflammatory Response Generated on Adenovirus Administration Is Markedly Attenuated When the Injection Is by IH Route. We analyzed IL-6, 
A
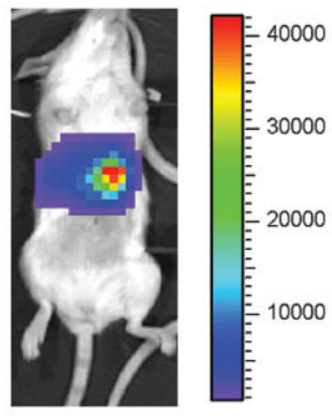

$\mathrm{IH}$

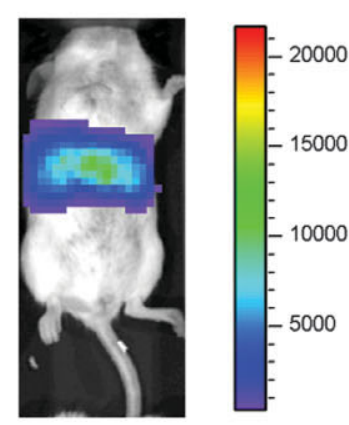

IV
C

IV

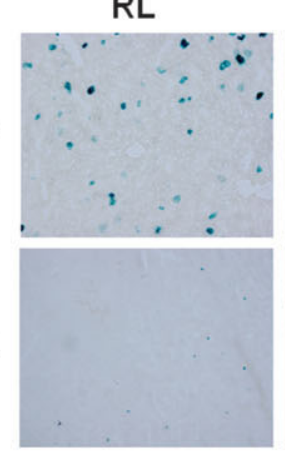

RML
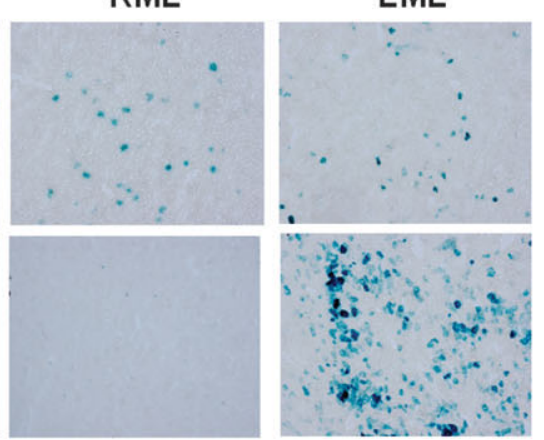

B

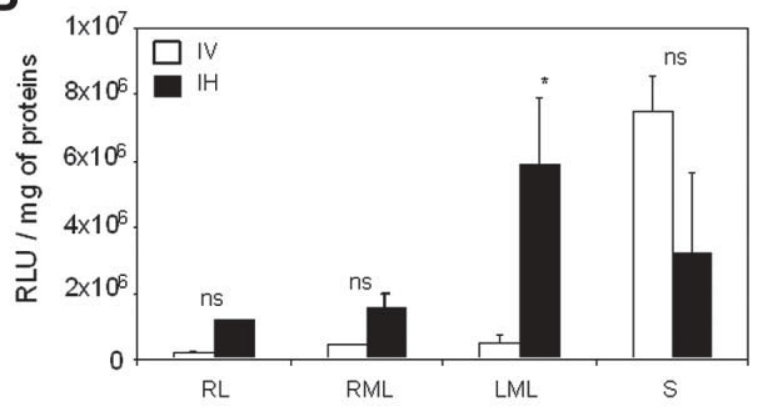

D

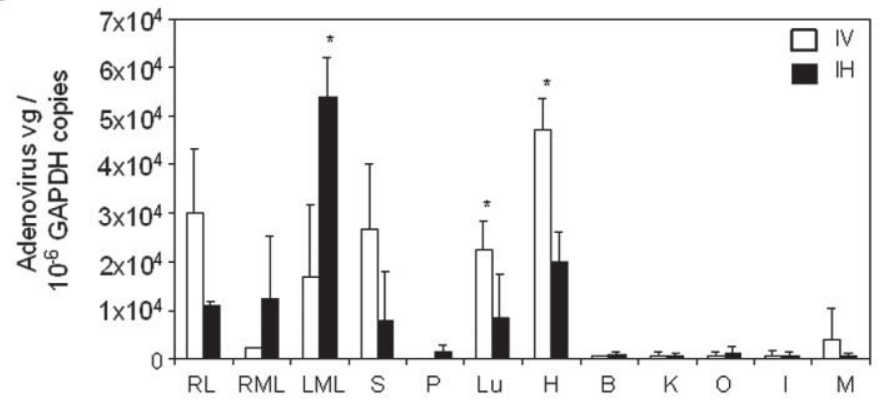

Fig. 2. In vivo and in vitro analysis of luciferase expression after intrahepatic (IH) or intravenous (IV) administration of Adluc and AdLacZ. (A) BALB/c mice $(n=3)$ were injected with $10^{10}$ pfu of Adluc by IH or IV routes. The picture corresponds to the image of luciferase expression 24 hours after injection and is representative of the results obtained in the rest of mice. (B) Thereafter, mice were killed to isolate the liver and the spleen. Luciferase expression was measured individually in the three major lobes of the liver: left medial lobe (LML), right medial lobe (injected lobe), and right lateral lobe and in the spleen by luminometry. Luciferase activity is expressed as RLU per milligram protein. Error bars represent \pm SD for triplicate measurements. (C) BALB/c mice $(n=3)$ were injected with $10^{10}$ pfu AdLacZ by IH or IV routes. Mice were sacrificed 24 hours after injection, and $\beta$-galactosidase expression was analyzed in the three major lobes of the liver. (D) Viral genomes (vg) were quantified in different organs and tissues: spleen (S), pancreas (P), lung (Lu), heart (H), brain (B), kidney (K), ovary (0), intestine (I), and muscle (M), 24 hours after IH or IV injection of $10^{10}$ pfu of AdLacZ, and normalized by $10^{-6} \mathrm{GAPDH}$ genome copies. Error bars represent \pm SD for triplicate measurements $\left({ }^{*} P<.05\right)$.

IL-12, and IFN- $\gamma$ serum levels in mice 6 and 24 hours after IH and IV injection of $10^{9}$ and $10^{10}$ pfu Adluc. As shown in Fig. 3A-C, high levels of IL-6, IL-12, and IFN- $\gamma$ were detected at 6 hours in serum from mice given IV injection of $10^{10} \mathrm{pfu}$ Adluc, and increased IL-6 values were observed in the serum of the animals injected IV with $10^{9}$ pfu. Surprisingly, mice receiving an IH dose of $10^{10} \mathrm{pfu}$ showed very low serum levels of the three cytokines, the values for all of them being undetectable in mice receiving the low dose of $10^{9} \mathrm{pfu}$, indicating the absence of inflammatory reaction in those animals.

Liver Injury. To determine vector-mediated hepatotoxicity, serum samples were analyzed for increases in the liver enzymes ALT and AST. Because of the biphasic nature of the ALT response to Ad administration, ${ }^{11}$ the levels of transaminases in serum was assayed at 6 and 24 hours and 7 and 10 days after adenovirus IH or IV injection. As shown in Fig. 3D-E, at 6 and 24 hours after injection, all mice that received the virus IV displayed ALT levels only slightly higher than or within the normal range. However, in mice injected $\mathrm{IH}$ with a viral dose of $10^{9}$ or $10^{10}$, serum transaminases showed a rapid and marked elevation at 6 hours, whereas no change in transaminases was detected in mice receiving IH $50 \mu \mathrm{L}$ saline (data not shown). The rapid upraise of transaminases was vector dose dependent and was short-lived, with a sharp decline 24 hours after vector administration. During the second phase of liver injury at days 7 and 10 after adenovirus injection, ALT and AST levels were significantly higher than in control animals in the groups of mice receiving $10^{10} \mathrm{pfu}$ virus independently of the route of vector injection.

Direct Intrabepatic Administration of Adenovirus Vector Avoids Kupffer Cell Engulfment. Liver macrophages had been shown to engulf a high percentage of adenoviral particles when they are intravenously injected. ${ }^{14-18}$ To test the influence of macrophages on liver transduction after IH administration, 24 hours after clodronate-loaded liposomes injection, mice received either $10^{9}$ pfu Adluc or $10^{9}$ IU HC-Ad/RUhIL-12. Control 

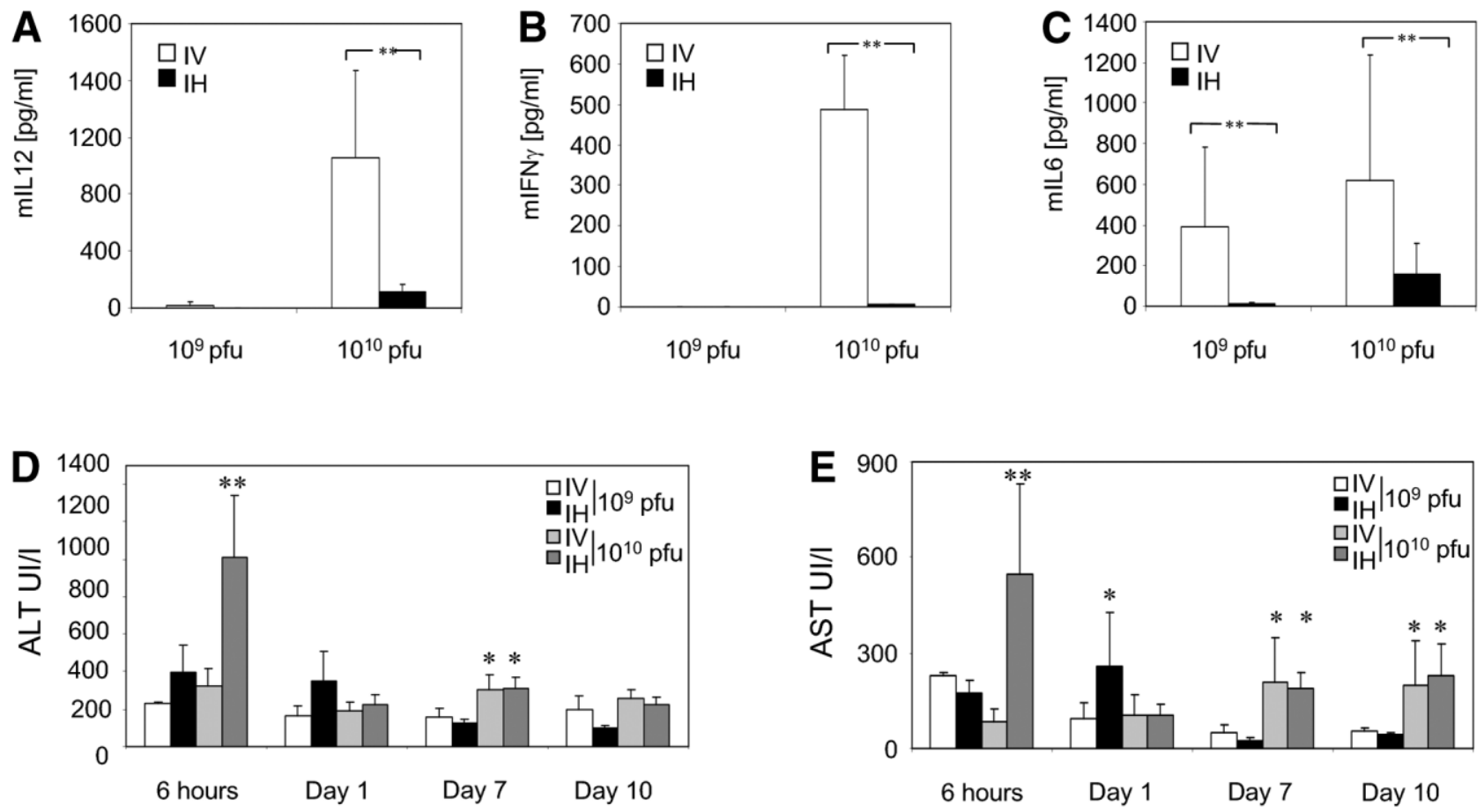

Fig. 3. Intrahepatic injection of adenovirus prevents systemic inflammatory reaction induced after intravascular injection. BALB/c mice were injected with Adluc by IH or IV route at a dose of $10^{9} \mathrm{pfu}$ or $10^{10} \mathrm{pfu} /$ mouse. Six hours after injection, serum was extracted, and concentrations of IL-12 (A), IFN- $\gamma(B)$, and IL-6 (C) were determined and presented as means of the values obtained from five mice. (D-E) Six hours and 1, 7, and 10 days after adenovirus injection, serum were extracted from the same mice, and transaminase levels were determined. Data are averages of five mice in each group. Error bars represent means \pm SD. Data from one of two experiments are provided.

mice received empty liposomes or saline. Luciferase expression was analyzed 3 days after vector administration, and hIL-12 was analyzed 10 hours after the administration of RU486 at a dose of $250 \mu \mathrm{g} / \mathrm{kg}$. As shown in Fig. $4 \mathrm{~A}-\mathrm{B}$, and in agreement with previous reports, ${ }^{14-18}$ a significant increase in transgene expression was observed after macrophage depletion, when mice received IV Adluc or Ad/RUhIL-12. Injection of empty liposomes also resulted in a marked increase in transgene expression, although not to the same levels. After IH injection of the vector, transgene expression was also increased by pretreatment with empty liposomes; however, the administration of clodronate-loaded liposomes did not further improve transduction. Interestingly, the increment on transgene expression attributable to macrophage depletion was significantly lower after IH than after IV injection. Analysis of liver sections from mice treated with empty- or clodronate-loaded liposomes by immunofluorescence (Fig. 4C) using the macrophage marker F4/80 and quantification using the Aquacosmos software, showed that whereas liver macrophages completely disappear after injection of clodronate-loaded liposomes, they remain intact in the mice that have received empty liposomes or saline.
Intrahepatic Injection of a High-Capacity Adenovirus Expressing Liver-Specific and Regulable Murine IL-12 Enhances Its Antitumoral Efficacy. To determine the significance of our findings, we evaluate the antitumoral effect of a high-capacity adenovirus encoding murine IL-12 after intrahepatic injection. Thirty C57BL/6 mice were $\mathrm{IH}$ injected with $5 \times 10^{5} \mathrm{MC} 38$ cells. Three days later the mice were opened and tumor was measured to ensure tumor engraftment and equivalent tumor size in all groups before initiation of the treatment. One group $(n=10)$ received an IV injection of $2 \times$ $10^{8} \mathrm{IU}$ of an HC-adenovirus expressing mifepristone-inducible murine IL-12 (HC-Ad/RUmIL-12) in $200 \mu \mathrm{L}$ saline. A second group $(\mathrm{n}=10)$ received the same dose of HC-Ad/RUmIL-12 by IH route in a volume of $50 \mu \mathrm{L}$, and the third group received saline by IV $(n=5)$ or IH $(\mathrm{n}=5)$ route. Seven days later, the expression of IL-12 was induced by administration of $250 \mu \mathrm{g}$ RU486 per kilogram body weight intraperitoneally during 10 consecutive days. mIL-12 and mIFN- $\gamma$ expression in serum was measured 10 hours after the first and fifth induction by ELISA. No IL-12 or IFN- $\gamma$ were detected in the serum of animals treated with IH or IV saline. As shown in Fig. 5, the expression of both cytokines was significantly higher 

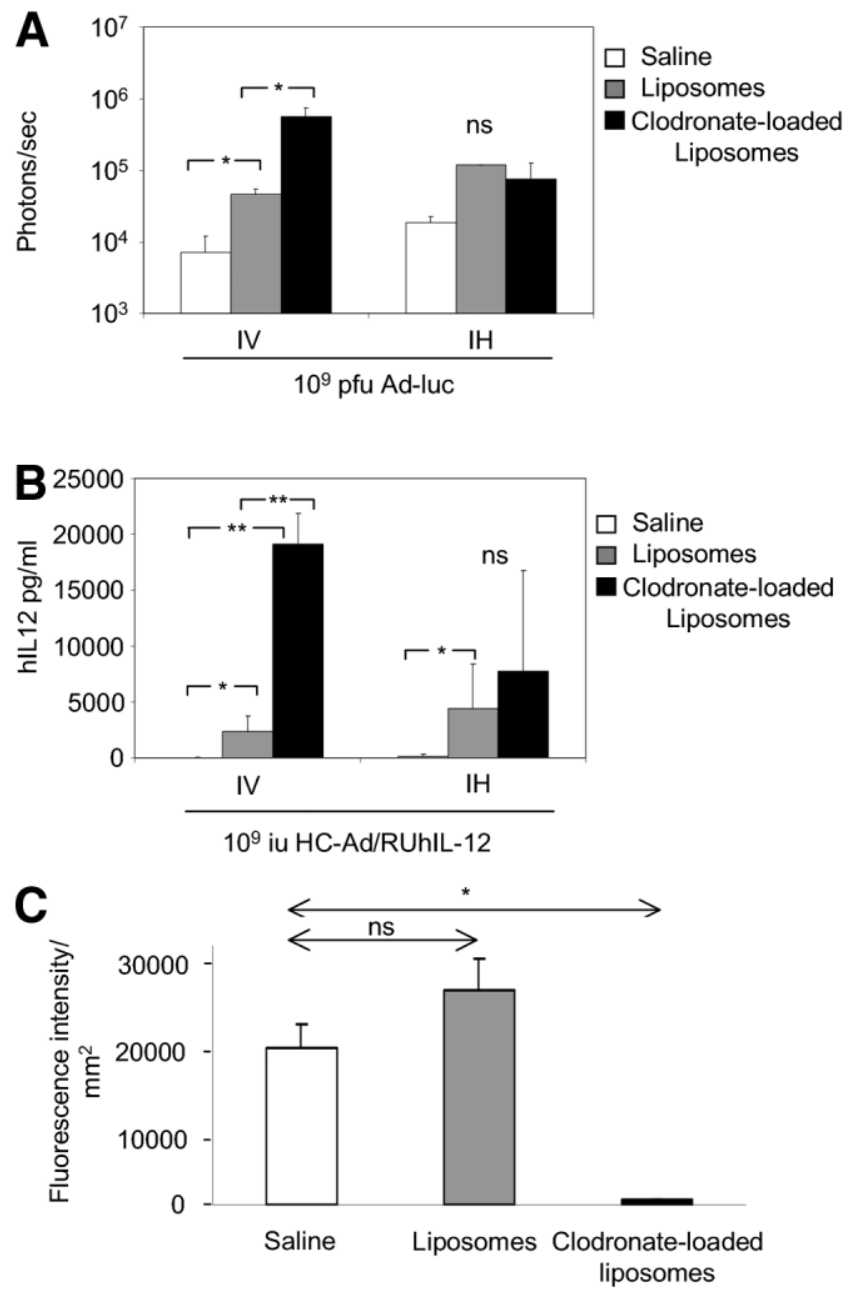

Fig. 4. Intrahepatic (IH) administration partially overcomes macrophage phagocytic activity. BALB/c mice were treated IV with saline solution (saline), PBS-liposomes (liposomes), or clodronate-loaded liposomes (clodronate). Twenty-four hours later, the animals received IV or IH, $10^{9}$ pfu of Adluc (A), or $10^{9}$ iu HD-AdhIL12 (B). (A) Three days after Adluc injection, luciferase expression was analyzed and quantified camera and presented as mean of the values obtained from six mice. (B) Two weeks after HD-AdhIL12 injection, hlL-12 expression was induced by a single administration of $250 \mu \mathrm{g} / \mathrm{kg}$ animal weight of RU486, and blood samples were collected 10 hours later. The concentration of hIL-12 was determined and is represented as mean \pm SD of the values obtained from three mice. $\left({ }^{*} P<.05\right)(C)$ Mice receiving saline, empty liposomes, or clodronate-loaded liposomes via tail vein were killed 24 hours later, and the presence of liver macrophages was analyzed by immunofluorescence using an anti-F4/80 antibody. Fluorescence signal was quantified and represented as fluorescence intensity per $\mathrm{mm}^{2}$.

in the group of mice receiving the virus IH. Two of the mice that received the virus $\mathrm{IH}$ died during the period of induction. These two mice showed the highest levels of IL-12 and IFN- $\gamma$ after the fifth induction. They showed a rapid weight loss but no liver tumor or pulmonary metastasis, suggesting that the death was caused by IL-12 toxicity.

Thirty days after tumor induction, tumor volume was measured in all mice by laparotomy. Because no differ- ences were found between the animals receiving saline by IH or IV administration, they were grouped as control group. As shown in Fig. 5C, the tumor sizes in the group of mice that received the vector $\mathrm{IH}$ were significantly smaller than in the group that received the vector via tail vein $(P=.038)$. Furthermore, the survival rate of the mice was also improved in animals that received the vector using the IH route compared with IV administration $(P=.02)$ (Fig. 5D).

\section{Discussion}

Adenovirus-based liver-directed gene therapy is frequently performed by injecting the vector intravascularly. By this route, a high amount of viral particles is needed to achieve a significant level of hepatocyte transduction. This is a main limitation for clinical application, because the administration of high doses of adenovirus is associated with a strong systemic inflammatory reaction that can be very severe and even fatal. ${ }^{11-13}$ Several strategies have been tested to reduce adenovirus-mediated inflammatory reaction without losing transduction efficiency. These include modification of vector capsid, ${ }^{35,36}$ administration of anti-inflammatory or immunosuppression agents, ${ }^{37,38}$ and modification of the route of administration. ${ }^{25}$

When the adenovirus vector is given intravascularly, the viral particles enter the liver sinusoids, where they encounter Kupffer cells, which engulf most of the virions. ${ }^{14}$ Vector uptake by Kupffer cells has two important consequences: first, it results in a reduction of liver transduction, especially relevant when the vector dose is low, ${ }^{14,15}$ and second, Kupffer cells are activated and release proinflammatory cytokines, including IL-6 and IL12. ${ }^{19-21}$ The latter induces the synthesis of IFN- $\gamma$, which contributes importantly to the toxicity of the vector. ${ }^{20}$

We obtained higher transduction efficiency by IH than by IV route of Ad administration. Our analysis on the effect of macrophage depletion on liver transduction after $\mathrm{IH}$ and IV adenovirus injection showed that the injection of the virus directly into the liver parenchyma partially overcomes macrophages phagocytic activity. Elimination of macrophages by using clodronate-loaded liposomes results in an increase in transgene expression after IH or IV injection. However, macrophage depletion increased transgene expression more markedly after IV injection than after IH administration. By this last route, differences in the level of transgene expression were not statistically significant after macrophage elimination. Of interest, pretreatment with empty liposomes does not deplete Kupffer cells but may temporarily block its phagocytic activity increasing transduction of hepatocytes. Thus, the use of empty liposomes might be an option for 

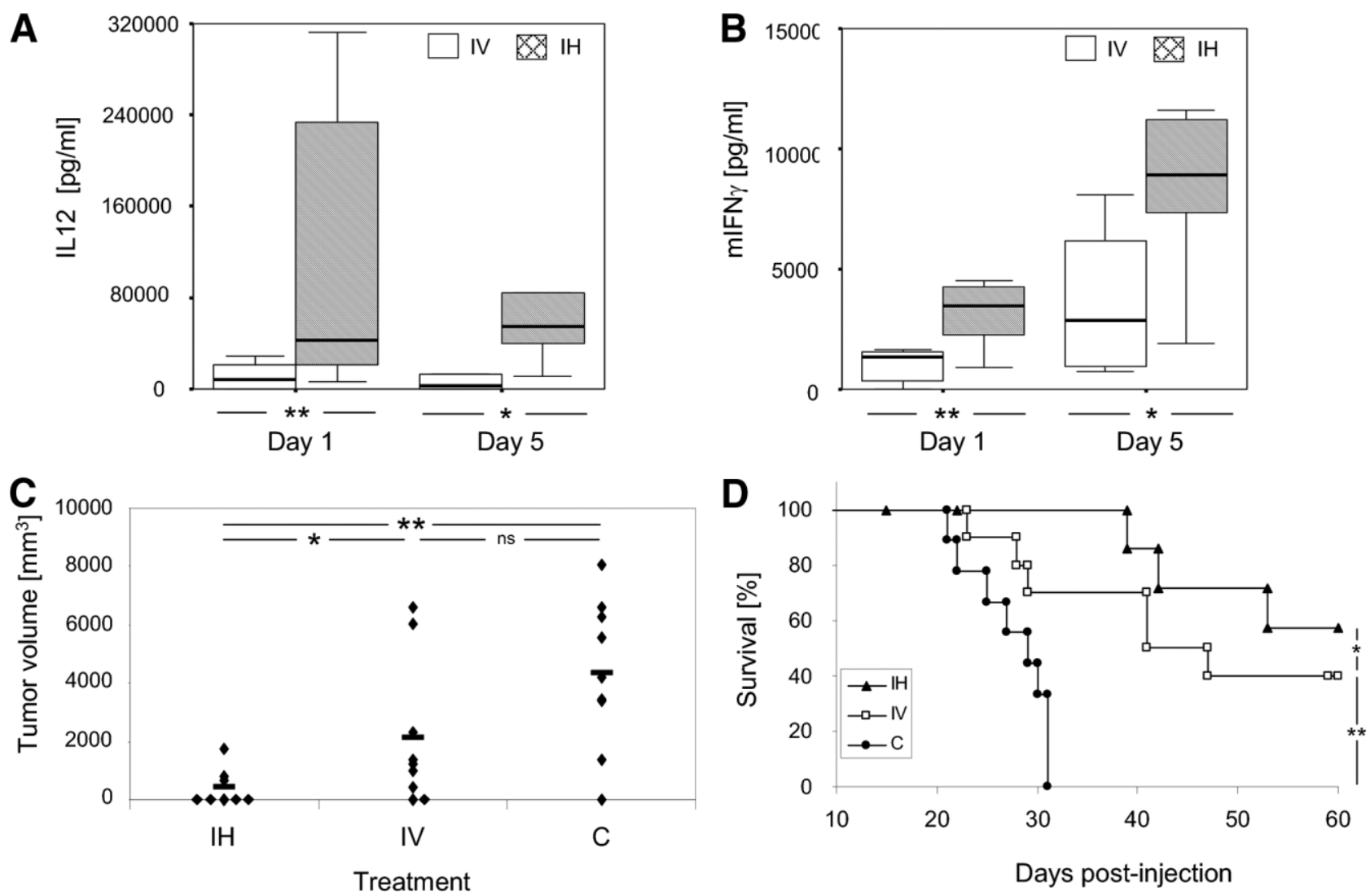

Fig. 5. Intrahepatic $(\mathrm{IH})$ administration of HC-Ad/RUmlL-12 results in an enhancement of antitumoral activity. Thirty C57BL/ 6 mice were injected IH with $5 \times 10^{5} \mathrm{MC} 38$. Three days later, tumor volume was measured, and mice were separated into three groups of 10 mice according to the treatment: $2 \times 10^{8}$ iu HC-Ad/RUmIL-12 IV, $2 \times 10^{8}$ iu HC-Ad/RUmIL-12 IH, and saline solution IV or IH. After 10 days, IL-12 expression was induced daily for 10 days by intraperitoneal administration of RU486 (250 $\mu \mathrm{g} / \mathrm{kg}$ animal weight). Peripheral blood samples were collected 10 hours after the first and fifth induction, and (A) IL-12 and (B) IFN- $\gamma$ were analyzed by ELISA. Data are presented in box-plot and were compared using the Mann-Whitney test. (C) Thirty days after cell injection, tumor volume was measured in all the mice after laparotomy, and values from individual mice were represented. (D) Kaplan-Meier survival analysis of mice with established MC38 tumors after treatment is assessed $\left({ }^{*} P<.05 ; * * P<.01\right)$.

certain clinical applications of liver-directed gene therapy. ${ }^{39}$

Two main consequences result from overcoming macrophage phagocytic activity by injecting the adenoviral vector directly into the hepatic parenchyma. First, the $\mathrm{IH}$ route leads to a significant increase of adenovirus-mediated liver transduction, which is especially important when a low dose of virus is administered. Second, and very importantly, IH administration markedly reduces the secretion of proinflammatory cytokines in serum. The reduction of the systemic inflammatory response in association with augmented transduction efficiency is an advantage of IH leading to increased efficacy with attenuated toxicity. Furthermore, the inflammatory response induced shortly after IV administration of adenoviral vectors has been shown to slow transgene expression. ${ }^{40}$ Thus, the lower inflammatory reaction on $\mathrm{IH}$ injection might explain the earlier peak of transgene expression when using this route of vector administration.
IH injection resulted in a more restricted distribution of the vector, which may contribute to lessening the systemic inflammatory reaction. Apart from the liver, the spleen is the organ most affected by adenovirus infection. ${ }^{10}$ Our data of luciferase expression and adenovirus distribution comparing IH and IV injections showed that the spread of the virus to the spleen and to other organs such as heart and lung is diminished after IH administration of the vector. Within the liver, IH injection of Ad allowed concentration of the expression of transgenic gene product at the site of injection. This may be advantageous for some clinical applications, as when treating tumor nodules in which long-term perinodular expression of IL-12 is desirable.

Adenoviral vectors are associated with a biphasic profile in liver injury. ${ }^{41}$ Although the second wave of hepatocellular damage is similar after IH or IV administration, we observed that hypertransaminasemia occurring few hours after IH vector injection was dose related and was 
higher than when using the IV route. Because Ad given by IH injection may partially escape Kupffer cells, it seems possible that, when using this route, hypertransaminasemia may reflect a higher number of virions entering hepatocytes. This toxicity is rapidly reversible, being over after 24 hours, and does not affect survival except when the expressed transgene has an intrinsic toxic effect such as murine IL-12 in mice.

To determine the significance of our findings, we evaluated the antitumoral efficacy of a high-capacity adenovirus encoding murine IL-12 given by IH route. In previous reports, we showed that first-generation and high-capacity adenovirus vectors expressing of murine IL-12 displayed significant antitumor effects in animal models of liver cancer when given intravenously. ${ }^{13}$ Our current work shows that intrahepatic injection of a highcapacity adenovirus clearly resulted in improved antitumor efficacy. This enhancement of antitumor activity was associated with a higher production of IL-12 and IFN- $\gamma$ in animals treated by the $\mathrm{IH}$ route.

In summary, our data demonstrate that IH injection of adenoviral vectors reduces adenovirus-mediated systemic inflammatory response and increases transgene expression by avoiding macrophage uptake. Moreover, we show that the antitumoral effect of an HC-Ad encoding IL-12 is boosted when given the vector by $\mathrm{IH}$ route. In clinical practice, intrahepatic injection of Ad can be performed safely under ultrasound guidance, ${ }^{27}$ and the IH route therefore should be considered in the design of gene therapy clinical trials.

Acknowledgment: The authors thank Celia Gomar, Paula Garces, Africa Vales, and Cristina Olagüe for technical assistance and Matilde Bustos and Eduardo Martínez-Ansó for professional technical assistance and useful suggestions.

\section{References}

1. Jaffe HA, Danel C, Longenecker G, Metzger M, Setoguchi Y, Rosenfeld $\mathrm{MA}$, et al. Adenovirus-mediated in vivo gene transfer and expression in normal rat liver. Nat Genet 1992;1:372-378.

2. Horowitz J. Adenovirus-mediated p53 gene therapy: overview of preclinical studies and potential clinical applications. Curr Opin Mol Ther 1999; 1:500-509.

3. Everts M, Curiel DT. Transductional targeting of adenoviral cancer gene therapy. Curr Gene Ther 2004;4:337-346.

4. Breyer B, Jiang W, Cheng H, Zhou L, Paul R, Feng T, et al. Adenoviral vector-mediated gene transfer for human gene therapy. Curr Gene Ther 2001;1:149-162.

5. Vorburger SA, Hunt KK. Adenoviral gene therapy. Oncologist 2002;7:4659.

6. Kochanek S, Schiedner G, Volpers C. High-capacity "gutless" adenoviral vectors. Curr Opin Mol Ther 2001;3:454-463.

7. Palmer DJ, Ng P. Helper-dependent adenoviral vectors for gene therapy. Hum Gene Ther 2005;16:1-16.
8. Muruve DA, Cotter MJ, Zaiss AK, White LR, Liu Q, Chan T, et al. Helper-dependent adenovirus vectors elicit intact innate but attenuated adaptive host immune responses in vivo. J Virol 2004;78:5966-5972.

9. Schiedner G, Morral N, Parks RJ, Wu Y, Koopmans SC, Langston C, et al. Genomic DNA transfer with a high-capacity adenovirus vector results in improved in vivo gene expression and decreased toxicity. Nat Genet 1998; 18:180-183.

10. Zhang Y, Chirmule N, Gao GP, Qian R, Croyle M, Joshi B, et al. Acute cytokine response to systemic adenoviral vectors in mice is mediated by dendritic cells and macrophages. Mol Ther 2001;3:697-707.

11. Varnavski AN, Calcedo R, Bove M, Gao G, Wilson JM. Evaluation of toxicity from high-dose systemic administration of recombinant adenovirus vector in vector-naive and pre-immunized mice. Gene Ther 2005;12: 427-436.

12. Raper SE, Yudkoff M, Chirmule N, Gao GP, Nunes F, Haskal ZJ, et al. A pilot study of in vivo liver-directed gene transfer with an adenoviral vector in partial ornithine transcarbamylase deficiency. Hum Gene Ther 2002; 13:163-175.

13. Raper SE, Chirmule N, Lee FS, Wivel NA, Bagg A, Gao GP, et al. Fatal systemic inflammatory response syndrome in a ornithine transcarbamylase deficient patient following adenoviral gene transfer. Mol Genet Metab 2003;80:148-158.

14. Tao N, Gao GP, Parr M, Johnston J, Baradet T, Wilson JM, et al. Sequestration of adenoviral vector by Kupffer cells leads to a nonlinear dose response of transduction in liver. Mol Ther 2001;3:28-35.

15. Ziegler RJ, Li C, Cherry M, Zhu Y, Hempel D, van Rooijen N, et al. Correction of the nonlinear dose response improves the viability of adenoviral vectors for gene therapy of Fabry disease. Hum Gene Ther 2002;3: 935-945.

16. Kuzmin AI, Finegold MJ, Eisensmith RC. Macrophage depletion increases the safety, efficacy and persistence of adenovirus-mediated gene transfer in vivo. Gene Ther 1997;4:309-316.

17. Stein CS, Pemberton JL, van Rooijen N, Davidson BL. Effects of macrophage depletion and anti-CD40 ligand on transgene expression and redosing with recombinant adenovirus. Gene Ther 1998;5:431-439.

18. Schiedner G, Hertel S, Johnston M, Dries V, van Rooijen N, Kochanek S. Selective depletion or blockade of Kupffer cells leads to enhanced and prolonged hepatic transgene expression using high-capacity adenoviral vectors. Mol Ther 2003; 7:35-43.

19. Liu Q, Muruve DA. Molecular basis of the inflammatory response to adenovirus vectors. Gene Ther 2003;10:935-940.

20. Lieber A, He CY, Meuse L, Schowalter D, Kirillova I, Winther B, et al. The role of Kupffer cell activation and viral gene expression in early liver toxicity after infusion of recombinant adenovirus vectors. J Virol 1997;71: 8798-8807.

21. Muruve DA, Barnes MJ, Stillman IE, Libermann TA. Adenoviral gene therapy leads to rapid induction of multiple chemokines and acute neutrophil-dependent hepatic injury in vivo. Hum Gene Ther 1999;10:965-976.

22. Huard J, Lochmuller H, Acsadi G, Jani A, Massie B, Karpati G. The route of administration is a major determinant of the transduction efficiency of rat tissues by adenoviral recombinants. Gene Ther 1995;2:107-115.

23. Nunes FA, Furth EE, Wilson JM, Raper SE. Gene transfer into the liver of nonhuman primates with E1-deleted recombinant adenoviral vectors: safety of readministration. Hum Gene Ther 1999;10:2515-2526.

24. Sullivan DE, Dash S, Du H, Hiramatsu N, Aydin F, Kolls J, et al. Liverdirected gene transfer in non-human primates. Hum Gene Ther 1997;8: 1195-1206.

25. Peeters MJ, Patijn GA, Lieber A, Meuse L, Kay MA. Adenovirus-mediated hepatic gene transfer in mice: comparison of intravascular and biliary administration. Hum Gene Ther 1996;7:1693-1699.

26. Gerolami R, Cardoso J, Lewin M, Bralet MP, Sa Cunha A, Clement O, et al. Evaluation of HSV-tk gene therapy in a rat model of chemically induced hepatocellular carcinoma by intratumoral and intrahepatic artery routes. Cancer Res 2000;60: 993-1001.

27. Sangro B, Mazzolini G, Ruiz J, Herraiz M, Quiroga J, Herrero I, et al. Phase I trial of intratumoral injection of an adenovirus encoding interleukin-12 for advanced digestive tumors. J Clin Oncol 2004;22:1389-1397. 
28. Lipshutz GS, Flebbe-Rehwaldt L, Gaensler KM. Adenovirus-mediated gene transfer to the peritoneum and hepatic parenchyma of fetal mice in utero. Surgery 1999;126:171-177.

29. Wood M, Perrotte P, Onishi E, Harper ME, Dinney C, Pagliaro L, et al. Biodistribution of an adenoviral vector carrying the luciferase reporter gene following intravesical or intravenous administration to a mouse. Cancer Gene Ther 1999;6:367-372.

30. Wang L, Hernandez-Alcoceba R, Shankar V, Zabala M, Kochanek S, Sangro B,et al. Prolonged and inducible transgene expression in the liver using gutless adenovirus: a potential therapy for liver cancer. Gastroenterology 2004;126:278-289.

31. Van Rooijen N, Sanders A. Liposome mediated depletion of macrophages: mechanism of action, preparation of liposomes and applications. J Immunol Methods 1994;174:83-93.

32. Van Rooijen N, Sanders A, van den Berg TK. Apoptosis of macrophages induced by liposome-mediated intracellular delivery of clodronate and propamidine. J Immunol. Methods 1996;193:93-99.

33. Winer J, Jung CK, Shackel I, Williams PM. Development and validation of real-time quantitative reverse transcriptase-polymerase chain reaction for monitoring gene expression in cardiac myocytes in vitro. Anal Biochem 1999;270:41-49.

34. Wu JC, Sundaresan G, Iyer M, Gambhir SS. Noninvasive optical imaging of firefly luciferase reporter gene expression in skeletal muscles of living mice. Mol Ther 2001;4:297-306.
35. Snoeys J, Mertens G, Lievens J, van Berkel T, Collen D, Biessen EA, et al. Lipid emulsions potently increase transgene expression in hepatocytes after adenoviral transfer. Mol Ther 2006;13:98-107.

36. Mok H, Palmer DJ, Ng P, Barry MA. Evaluation of polyethylene glycol modification of first-generation and helper-dependent adenoviral vectors to reduce innate immune responses. Mol Ther 2005;11:6679.

37. Hermens WT, Verhaagen J. Suppression of inflammation by dexamethasone prolongs adenoviral vector-mediated transgene expression in the facial nucleus of the rat. Brain Res Bull 1998;47:133-140.

38. Smith TA, White BD, Gardner JM, Kaleko M, McClelland A. Transient immunosuppression permits successful repetitive intravenous administration of an adenovirus vector. Gene Ther 1996;3:496-502.

39. Park JW, Benz CC, Martin FJ. Future directions of liposome- and immunoliposome-based cancer therapeutics. Semin Oncol 2004;31: 196-205.

40. Schnell MA, Zhang Y, Tazelaar J, Gao GP, Yu QC, Qian R, et al. Activation of innate immunity in nonhuman primates following intraportal administration of adenoviral vectors. Mol Ther 2001;3: 708-722.

41. Liu Q, Zaiss AK, Colarusso P, Patel K, Haljan G, Wickham TJ, et al. The role of capsid-endothelial interactions in the innate immune response to adenovirus vectors. Hum Gene Ther 2003;14:627-643. 